\title{
Understanding Female-Focused Hackathon Participants' Collaboration Styles and Event Goals
}

\author{
Brittany Ann Kos \\ ATLAS Institute, University of Colorado Boulder \\ Boulder, $\mathrm{CO}$ \\ brittany.kos@colorado.edu
}

\begin{abstract}
Collegiate hackathons are informal learning environments where students build new projects in a limited amount of time and often compete for prizes. Previous hackathon research has shown that students go to these events to learn new technical skills, work on projects with friends, or network with the technical community; however, little is known about the different ways in which students collaborate with other participants and how students' event goals affect their trajectory at the hackathon. In the literature, students are typically described as working in competitive teams that build one project to be entered into a competition. However, there are gaps when it comes to discussing other ways students may work together or what other non-competitive projects/activities students may want to engage with at a hackathon. This paper reports on different collaboration styles and event goals documented at a Spring 2018 female-focused hackathon. Findings include three types of collaboration styles: team-based, cooperative group, and individual participation; as well as four types of event goals: competition, exploration, dabbling, and observing. These findings can inform the collegiate hackathon literature and help hackathons diversify their events by offering insight into ways diverse participants choose to participate at a hackathon and offering design suggestions that mainstream collegiate hackathons can adopt to be more inclusive of different types of students.
\end{abstract}

\section{CCS CONCEPTS}

- Social and professional topics $\rightarrow$ Informal education; Women; - Applied computing $\rightarrow$ Collaborative learning.

\section{KEYWORDS}

hackathon, informal learning, teamwork, women

\section{ACM Reference Format:}

Brittany Ann Kos. 2019. Understanding Female-Focused Hackathon Participants' Collaboration Styles and Event Goals. In International Conference on Game Jams, Hackathons and Game Creation Events 2019 (ICGF 2019), March 17, 2019, San Francisco, CA, USA. ACM, New York, NY, USA, 4 pages. https://doi.org/10.1145/3316287.3316292

Permission to make digital or hard copies of all or part of this work for personal or classroom use is granted without fee provided that copies are not made or distributed for profit or commercial advantage and that copies bear this notice and the full citation on the first page. Copyrights for components of this work owned by others than the author(s) must be honored. Abstracting with credit is permitted. To copy otherwise, or republish, to post on servers or to redistribute to lists, requires prior specific permission and/or a fee. Request permissions from permissions@acm.org.

ICG7 2019, March 17, 2019, San Francisco, CA, USA

(C) 2019 Copyright held by the owner/author(s). Publication rights licensed to ACM. ACM ISBN 978-1-4503-6205-4/19/03...\$15.00

https://doi.org/10.1145/3316287.3316292

\section{INTRODUCTION AND BACKGROUND}

Student hackathons are a specific type of hackathon, aimed at college students, recent college graduates, and sometimes high school students. These are informal learning environments where students compete against each other in a project-building marathon, usually 24-to-36-hours long, held on a university campus, and open-ended project-building competitions. Participants can create software or hardware projects, called "hacks," and optionally compete for prizes [13]. Students attend these events because they provide an opportunity for informal learning, networking, and building products for social change[4].

Teamwork: Most of the literature on hackathon teams has focused on how teams form and collaborate together. Due to the brief and intense nature of hackathons, negotiating the responsibilities of teamwork are important to the project's success [12]. Teams are non-permanent, and often, participants will switch or re-form teams during the hackathon [6]. Technological solutions have been suggested that help participants form teams, organize projects, and keep in communication with each other [3]; however, these technologies are not widely used at student hackathons. There is little research done on non-team form of participation at hackathons.

Event Goals: There has been little research on participant's event goals. There have been a number of hackathon studies researching student motivations for attending hackathon events and they show a wide range of motivating factors. Many students have listed other motivations for attending the hackathon, such as learning [1, 4, 6, 9-13], networking [4, 10], and teamwork [10, 11, 13]. Competition is a less popular motivation, often ranking behind the more social aspects of a hackathon, like working with friends or in teams [4]. However, there have been few studies or event reports that address how participants might interact with the event based on their different motivations. There is little known about how the participants might want to engage with the event (e.g. if they want to participate in the competition; if they want to attend workshops; if they want to work in a team; etc) - what this paper calls "event goals".

Competition: Competition is a common element of mainstream hackathons where there can be multiple contests held at a time at one event, such as national contests (e.g. the continental leagues hosted by Major League Hacking [7], placement contest (e.g. 1st, 2nd, and 3rd place winners), and sponsor contests (e.g. "Best Use of Company Foo's API"). Competition can be a motivating factor for some students [13]. Still, a hackathon's competitive design has some negative effects: it is more difficult to foster open collaboration in a competitive environment [11]; it stifles creativity and free exploration [5]; it positions participants against one another [11]; and it can deter students from attending due to a perceived overly 
competitive atmosphere [13]. Participating in competition is one of the only only event goals that the hackathon literature discusses and there is little other discussion about participants who are not motivated by competition.

Female-Focused Hackathons: Similar to the computing field overall, there is a disproportionate number of men attending hackathons over women. One survey of industry and open (i.e. non-student) hackathons, only $11 \%$ of attendees were women [2] and student hackathons only average of $28 \%$ female attendance [8]. However, these numbers are still low. There has been some work trying to diversify hackathons and expand to a larger audience [11], and some organizers have suggestions for how to change a hackathon environment to be more welcoming female students $[9,10]$. The student hackathon community has also responded to this problem by creating women-only, female-focused, and gender equal hackathons. However, most of the studies conducted about hackathons have been on "mainstream" (i.e. non-gender focused) hackathons.

Gaps: There are gaps in the hackathon literature about nonteam participation at hackathons and the literature expresses the idea that community, teamwork, and competition are important elements of hackathons without addressing participants who work individually, as well as how . There is also a lack of research and experience reports about female-focused hackathons. This event report paper will address the gaps we see in literature and within the student hackathon community.

This paper discusses preliminary findings about the different collaboration styles and event goals of the participants of a femalefocused hackathon. Findings from this event report can be used to (1) inform our knowledge of female-focused hackathon participants; (2) expand the hackathon literature on collaboration and event goals; and (3) inform the inclusion practices of all (mainstream and female-focused) student hackathons.

\section{METHODOLOGY}

In the spring of 2018, the author of this paper worked as the lead organizer of a women and non-binary hackathon, called T9Hacks. This paper reports on the different "patterns of participation" the T9Hacks team observed at the event. In the Discussion section, this paper discusses the implications that this new participation schema has on female-focused hackathons, the student hackathon literature, and designing hackathons for diversity.

\subsection{About T9Hacks}

T9Hacks is a student women and non-binary hackathon hosted by a student group at a large state university in the Southwest region of the United States. The spring 2018 event was the thirdannual event that was hosted on the same campus. T9Hacks a female-focused event, which means that registration did not exclude cisgender men ${ }^{1}$; however the hackathon was designed to create an inclusive and welcoming event for women and non-binary students specifically ${ }^{2}$. The spring 2018 event had 136 total participants, with

\footnotetext{
1 "Cisgender" or simply "cis" refers to an individual whose gender matches their sex assigned at birth. For example, a person who was assigned male at birth, and who identifies as a man, is considered a cis-man.

${ }^{2}$ The paper uses the term "non-binary" as an umbrella term to refer to a student whose gender falls outside of the hegemonic binary (male-female). For example, a
}

$65 \%$ women, $5 \%$ non-binary and $30 \%$ men attending. For $64 \%$ of the hackers, this was their first hackathon.

Structurally, the event was very similar to other mainstream hackathons: it was a 24-hour student-only competitive hackathon. Before the event, participants registered for the hackathon, and possibly formed teams with classmates or friends. At the event, participants would finalize teams, or choose to work alone. Students would work on projects, attend workshops, and interact with other students at the event. At the end of the hackathon, some students participated in science-fair style "demos" where they would present their projects to judges and potentially win prizes.

T9Hacks is student-led and student-organized. The spring 2018 organizational team consisted of three undergraduates, one graduate student (the lead organizer and author of this paper), and a faculty-advisor. All team members were experienced with hackathons, most team members had attended hackathons previously and have worked on the organizing team for T9hacks in the past.

\subsection{Creation of the "Patterns of Participation"}

At the spring 2018 T9Hacks hackathon, every member of the T9Hacks team talked with participants at the hackathon, with the goal of collecting personal narratives and anecdotes from the participants' experiences at the hackathon. Other activities during the hackathon, like workshops and meals provided additional opportunity for the team to speak with participants. These informal discussions asked questions like; What project are you working on?, What inspired your project idea?, Have you worked with X technology before?, Who are your team members?, Have you been to a hackathon before?, or What made you come to T9Hacks?

After the hackathon, the T9Hacks team had a series of postevent meetings, discussing where the hackathon was successful and where it could be improved. One of the topics that was discussed heavily was the fact that there were types of participants at T9Hacks. These participants were motivated to attend the event for different reasons, had different skill levels with computing, and engaged with the event in different ways. As the team evaluated the event structure, it came about that these different participants could generally be motivated and deterred by the same activities. For example, some participants were excited to participate in the competition element of the hackathon, while others were intimidated by it and didn't want to compete.

The team began listing patterns they saw in the participants of the event: there were people who just came for the food, those who only came for workshops, those who wanted to explore on their own, those who came to work on personal projects, those who were excited about the competition, those who wanted to learn about a specific software or technology. Next, the team members who hosted the spring 2017 and spring 2016 events further refined this list by adding their participant observations from previous years. Finally, the author organized these patterns of participants into two themes that are covered in the student hackathon literature: collaboration and event goals.

genderqueer or agender student would be non-binary. This is congruent with how T9Hacks uses the term as well. 


\section{FINDINGS}

\subsection{Collaboration Styles}

This first theme focused on how the participants interacted with one another, collaborated on projects with each other, and the nature of the projects they created.

Traditional Teams: A traditional team is a group of students who work collaboratively on one project. Previous literature describes traditional teams as the expected way that students participate at hackathons. For example, a team may form at the beginning of the hackathon and don't collaborate with any other hackathon participants or teams. Teams on a single project throughout the event and showcase that project at demos.

Cooperative Group: Cooperative groups are formed by participants who are working on individual projects, but group with people working on similar projects to give each other mutual support. Participants in this category may call themselves a "team" because they were providing support and assistance to each other.

Individual Participation: Individual participation is when a participant works toward a single project goal, but without the support of either a traditional team or cooperative group. Though these students work alone, they can interact with other participants, however, these are usually social interactions as they work on their hackathon project by themselves.

\subsection{Event Goals}

The second theme focused on the difference in event goals that lead to different participant trajectories. This theme describes how the participants were motivated by different things, had different learning objectives from each other, and how these factors impacted which elements of the hackathon the participants interacted with.

Competition: This event goal is a typical way students are described at student hackathons. This describes someone (or a team of people) who are motivated by the spirit of competition. Their hackathon goal is to build a project that can be entered into one (or more) of the prize categories. Winning means both acquiring prizes and gaining recognition and positive reputation.

Exploration: In contrast, some participants are not motivated by competition, but are interested in the learning opportunities and time-constrained format a hackathon provides. An explorer may work on a "traditional" hackathon project (such as creating something with a newly learned technology or solving a problem), but they may also work on personal projects, such as a personal website or a project for a class. These students do not necessarily plan on participating in the competition aspect of the hackathon, but instead are driven by internal motivations.

Dabbling: The dabbler is similar to an explorer, but they are motivated to learn and explore many topics throughout the hackathon. They may not even work on one project throughout the hackathon, instead changing project ideas as they explore new areas of computing. Dabblers attend hackathons with the purpose of learning as much as they can in a limited amount of time.

Observing: Observers are non-participants at the hackathon; they are present at the event, but do not partake is many of the hackathon activities, such as competition, project building, or workshops. They may know another participant at the hackathon and are exposed to the computing elements of a hackathon second-hand.

\section{DISCUSSION}

Limitations and Strengths of the Sample Participants The participants who were used to create the groupings of participants for the findings were diverse in terms of gender, race, year in school, and major. All the participant information was anecdotal and presented in the T9Hacks post-event discussions as personal narratives. The results of this paper are not generalizable to other femalefocused or mainstream hackathons. This paper also does not make claims about certain demographic groups being more or less likely to engage in these different forms participation. However, what the T9Hacks team observed in spring 2018, was that students of color, especially women of color, as well as non-binary students participated heavily in the non-traditional means of participation.

Implications for hackathon literature The current hackathon literature implies that students who attend hackathons are motivated by competition and will work in teams, but there is a lack of discussion about the other ways students participate at a hackathon. This study observed these typical behaviors, as well as other collaboration styles and event goals. This exploratory study is a first pass at documenting and cataloging these experiences. Hackathon participation is not as straightforward as it has been presented in the past. Students choose to attend these events for different reasons that impact what activities they engage with at the event. Students also choose to work with other students in different ways, impacting the experiences and exposure each student has at these events.

This study is also one of the first female-focused hackathon studies. It gives a look into the collaboration styles and event goals of non-mainstream hackathon participants. More work needs to be done with female-focused hackathons to see if similar patterns of participation findings can be observed at similar hackathons. This research shows a range of ways students may choose to participate at hackathons. The findings can suggest that other participation types provide avenues for underrepresented students to explore a hackathon. The participant types presented in this paper are important to recognize in the hackathon literature, as well as in the hackathon community, since they provide additional trajectories that facilitate beginner, non-major, women, non-binary, or students or color engagement with the event.

Implications for diversifying hackathons The results of this study can be used to make student hackathons more inclusive to a wide range of participants. Though this data is exploratory, the participants in this sample are from diverse backgrounds in terms of gender, race, and hackathon experience. The sample suggests that there may be trends in students traditionally marginalized in computer science participating at the hackathon by using nonteam based collaboration methods and embracing non-competitive goals. At the spring 2018 event, most of the men were observed participating in teams, while less than half of the women and nonbinary participants chose the same. Though most of the hackathon participants were there to compete, there was a greater percentage of women and non-binary participants interested in exploring (and not competing) when compared to their male counter parts. These trends are compounded with intersection of race and ethnicity; though fewer women were competing than men, fewer students of color choose competition than their white peers. 
This work seeks to expand the resources available to student hackathon organizers on participant collaboration styles and event goals. The goal of this work is for it to be adopted and incorporated within the student hackathon community. Many of these participants were supported by the varying and flexible structural elements of the hackathon. Here are some design recommendations that can help create an inclusive environment for these different types of participants.

- Include different opportunities at the hackathon that will cater specifically to different collaboration styles. Do not require participants to work in teams and do not restrict team size.

- Include opportunities for participants with different event goals. Have different ways participants can engage with the event other than just a competition.

- Offer fun or low-stakes activities that all students can participate in. Build community at the hackathon by having activities that are not tied in with the content (competition, workshops, project building, etc.) of the hackathon. Students who have different event goals or who are working individually may not interact with each other if they are separated for the entire event. Professional development activities (resume reviews or taking professional headshots) or fun activities (cupcake decorating or morning yoga) can offer opportunities for all participants to meet each other and do activities that are not skill-based.

- Provide ways for participants to easily switch tracks or switch groups. Groups may change over the course of the event. The hackathon should be structured so that a participant can still engage with the event, even if their group, project, or topic may have changed.

- Encourage all participants to showcase their projects in demos, but if someone isn't interested, invite them to observe instead. Or create a separate non-competitive demo session for participants who are beginners or first timers to first get comfortable with demonstrating their project to others.

- Provide resources for beginners that will be accessible throughout the event. Make sure mentors are scheduled at all times (even in the night!), so someone is available to help at all times. Alternatively, schedule the hackathon to be a two-day event, so it will be easier to staff.

- Make it clear in the advertising that participants can leave the hackathon early if necessary. Pushing heavily on overnight attendance may deter some students from attending at all. Though some students may be interested or excited about attending a 24-hour or 36-hour event, for first-time hackathon participants, the timing of the hackathon may not be enticing.

- Allow for friends of the participants to attend the event, even if they are just observing and not directly engaging with the event. Allowing for friends and observers to attend the hackathon can help support the participants of the event, while potentially exposing more students to computing.

- Plan workshops and activities every day of the hackathon. This will give students who are exploring or dabbling an opportunity to participate throughout the run of the event.
If workshops are only hosted the first day or at the beginning of the event, some students may lose interest in the event and leave.

\section{CONCLUSION AND FUTURE WORK}

The different collaboration styles and event goals presented here in this paper can inform hackathon research and improve the design of hackathons. The design and structure of T9Hacks afforded ways the students could participate at the hackathon. The paper presents a list of design suggestions, taken from the design of T9Hacks, that offer ways to include these different types of participants. Future work includes further exploration on hackathon participant experiences. Follow-up studies can include replicating the study at another event, continuing the work to catalog different types of participants, or delving deeper into the experiences of hackathon participants and exploring how goals and collaboration differs across different demographic boundaries, such as gender, race, or major.

\section{ACKNOWLEDGMENTS}

The author would like to thank Simone Hyater-Adams, Kahlil B. Thomas, and Lecia Barker for their guidance, comments, and helpful suggestions.

\section{REFERENCES}

[1] Craig Anslow, John Brosz, Frank Maurer, and Mike Boyes. 2016. Datathons: An Experience Report of Data Hackathons for Data Science Education. In SIGCSE '16. ACM, New York, NY, USA.

[2] Gerard Briscoe and Catherine Mulligan. 2014. Digital Innovation: The Hackathon Phenomenon. Creativeworks London Working Paper. Creativeworks London.

[3] Adrienne S. Brown. 2016. Building Code, Building Relationships. Ph.D. Dissertation. Indiana University.

[4] Allan Fowler. 2016. Informal STEM Learning in Game Jams, Hackathons and Game Creation Events. In GfH\&GC '16. New York, NY, USA, 38-41.

[5] Lindsay Grace. 2016. Deciphering Hackathons and Game Jams Through Play. In GJH\&GC '16. ACM, New York, NY, USA, 42-45.

[6] Graham M. Jones, Beth Semel, and Audrey Le. 2015. "There's No Rules. It's Hackathon.": Negotiating Commitment in a Context of Volatile Sociality. Fournal of Linguistic Anthropology 25, 3 (2015), 322-345.

[7] Major League Hacking (MLH). 2018. About - Major League Hacking. https://mlh.io/about.

[8] Major League Hacking (MLH) and Shyamal Ruparel. 2015. Workshop: Hackathon Logistics.

[9] David Munro. 2015. Hosting Hackathons a Tool in Retaining Students with Beneficial Side Effects. F. Comput. Sci. Coll. 30, 5 (2015), 46-51.

[10] Arnab Nandi and Meris Mandernach. 2016. Hackathons As an Informal Learning Platform. In SIGCSE '16. ACM, New York, NY, USA, 346-351.

[11] Gabriela T. Richard, Yasmin B. Kafai, Barrie Adleberg, and Orkan Telhan. 2015. StitchFest: Diversifying a College Hackathon to Broaden Participation and Perceptions in Computing. In SIGCSE '15. ACM, New York, NY, USA, 114-119.

[12] Erik H. Trainer, Arun Kalyanasundaram, Chalalai Chaihirunkarn, and James D. Herbsleb. 2016. How to Hackathon: Socio-Technical Tradeoffs in Brief, Intensive Collocation. In CSCW' '16. ACM, New York, NY, USA, 1118-1130.

[13] Jeremy Warner and Philip J. Guo. 2017. Hack.Edu: Examining How College Hackathons Are Perceived By Student Attendees and Non-Attendees. In ICER '17. ACM, New York, NY, USA, 254-262. 